\title{
A Visualization Study of Mechanical Dispersion in Porous Media
}

\author{
Fujio Kuwahara*, Norimasa Sogawa and Takafumi Kuroiwa
}

Department of Mechanical Engineering, Shizuoka University, 3-5-1 Johoku, Hamamatsu, 432-8561 Japan

\begin{abstract}
A visualization technique using fluorescein sodium has been developed for the study of mechanical dispersion within fluid-saturated porous media. The technique is based on the correlation between the color intensity of blue and the concentration of fluorescein sodium. Having constructed the calibration curve to relate the blue intensity and the concentration, the measurements were made for the parallel streams of different concentration within the shallow channel where small rectangular blocks were arranged in a periodical fashion. The dilute fluorescein sodium solution was supplied from the central slot of the inlet section to form the central stream, at the same velocity as the adjacent water streams. The solution diffuses into these water streams as the central stream moves through the channel. The color intensity within the field of interest was monitored and then processed to obtain the concentration distribution. Numerical computation results based on the macroscopic mass transfer equation with an adjustable dispersion coefficient was compared against the concentration obtained from the visualization, so as to determine the dispersion coefficient, which provides the best agreement between the numerical and experimental results.
\end{abstract}

Keywords: Porous media, dispersion, mass transfer, visualization.

\section{INTRODUCTION}

Mechanical dispersion is the spreading of heat and species caused by variations in fluid velocity about the mean velocity. In addition to the molecular diffusion, there is significant mechanical dispersion in heat and mass in a fluidsaturated porous medium, as a result of hydrodynamic mixing of the fluid particles passing through pores. This mechanical dispersion causes additional heat and mass transfer, which arises further complications in dealing with transport processes in fluid saturated porous media. Exploiting a concentration visualization technique, we shall investigate the mechanical dispersion coefficient, which becomes predominant for convective flows in porous media.

According to Wakao and Kaguei [1], Yagi et al. [2] were the first to measure the effective longitudinal thermal conductivities of packed bed, taking full account of the effect of thermal dispersion, and eventually found the longitudinal component of the dispersion coefficient much greater than its transverse component. Since the famous analytical treatment in a tube by Taylor [3], a number of theoretical and experimental efforts (e.g. Aris [4], Koch and Brady [5], Han et al. [6], and Vortmeyer [7]) were made to establish useful correlations for estimating the effective thermal conductivities due to thermal dispersion (See Kaviany [8]). Furthermore, a series of numerical experiments were conducted by Kuwahara et al. [9] and Kuwahara and Nakayama [10], assuming a macroscopically uniform flow through a lattice of rods, so as to elucidate the effects of microscopic velocity and temperature fields on the thermal dispersion.

*Address correspondence to this author at the Department of Mechanical Engineering, Shizuoka University, 3-5-1 Johoku, Hamamatsu, 432-8561 Japan; Tel: +81-53-478-1046; Fax: +81-53-478-1046;

E-mail: tmfkuwa@ipc.shizuoka.ac.jp
In this experimental study, we shall focus on the effects of mechanical dispersion on the mass transfer diffusivity in liquid flow through a porous medium. The results obtained for mass transfer may readily be translated for the case of heat transfer or vice versa. Thus, We shall propose a novel visualization technique using a dilute fluorescein sodium solution, in order to determine the dispersion coefficient.

\section{Experimental Set-Up}

As shown in Fig. (1), shallow channels are constructed using acrylic plastic parallel plates. In one channel, rectangular blocks of acrylic plastic are placed in a regular fashion (Model 1), while in another channel, the blocks are arranged in a staggered fashion (Model 2), as shown in Figs. (2 (a) and (b)), respectively.

Water is steadily and uniformly supplied from three overflow tanks (3) into the channel of its height $3 \mathrm{~mm}$. The water in the central tank is saturated with fluorescein sodium. This fluorescein sodium solution flows into the channel at the same speed as the water streams from the two adjacent side tanks, as the pump (2) drives the streams. All three streams are rectified within a rectifying zone (5) to form a macroscopically single uniform stream within the channel. The fluorescein sodium solution occupying the central part of the stream diffuses into the adjacent water currents as the central stream travels through the channel.

The channel is uniformly lighted up from the bottom using the flat light panel (9)such that the miscible displacement in the test section (6) can be monitored by the camera (8) The Darcian velocity of the channel is measured by measuring the mass of the water flowing into the exit reservoir (7) per unit time. 


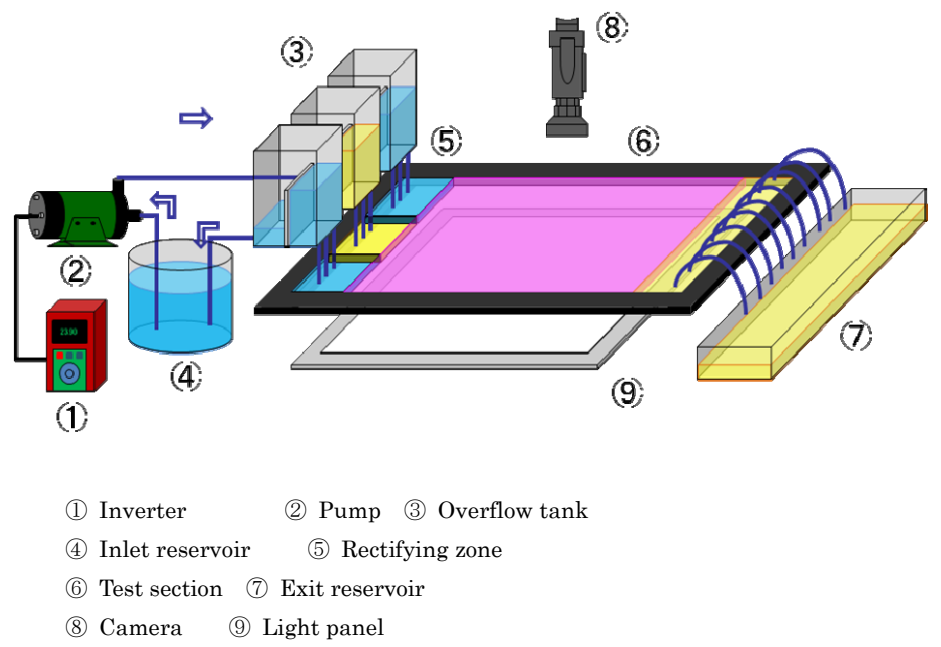

Fig. (1). Experimental apparatus.

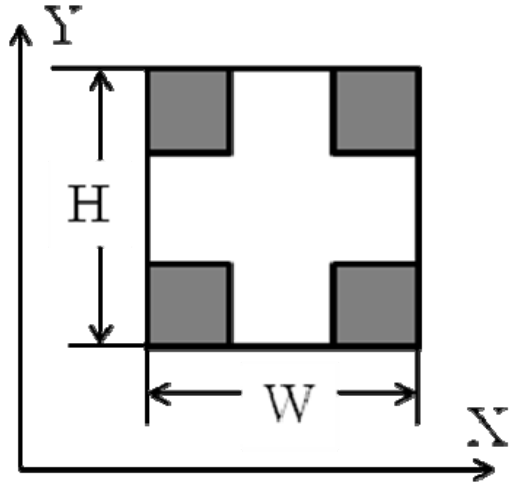

(a)Regular array

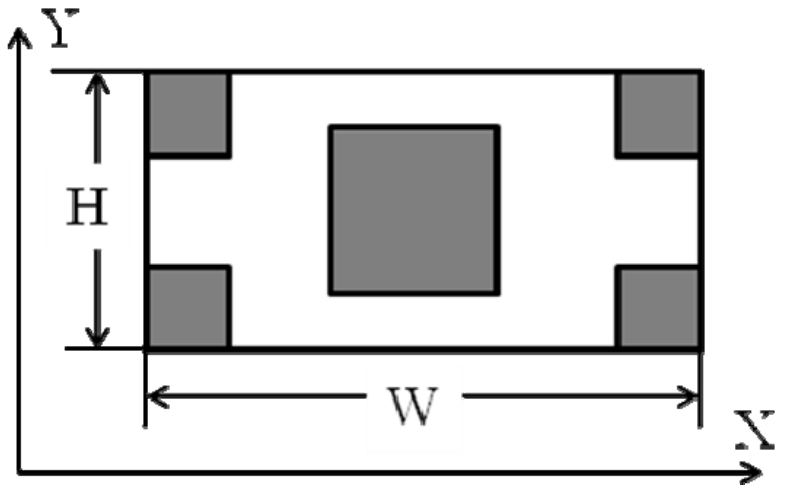

(b) Staggered array
Fig. (2). Physical models.

Two porosities $\varepsilon$, as shown in Table $\mathbf{1}$, are prepared for each arrangement of the square blocks in the channel by changing the size of the blocks.

The spatial development of the concentration field within the monitored section of the channel $(0.45 \mathrm{~m} \times 0.3 \mathrm{~m})$ as specifically illustrated in Fig. (3) is constantly videotaped from the top. These images are processed to obtain macroscopic solute concentration field. The Darcian velocity is varied from $1.5 \mathrm{~mm} / \mathrm{s}$ to $150 \mathrm{~mm} / \mathrm{s}$ to cover a wide range of Peclet number, roughly, from $10^{4}$ to $10^{6}$.

\section{Calculation of Concentration}

The blue intensity of the color indicators has been selected to determine the local solute concentration. Preliminary measurements using the solutions of prescribed concen-

Table. 1. Experimental Condition

\begin{tabular}{|c|c|c|c|c|}
\hline & \multicolumn{2}{|c|}{ Model 1 } & \multicolumn{2}{c|}{ Model 2 } \\
\hline \hline$D^{*}=D / H$ & 0.6 & 0.5 & 0.6 & 0.5 \\
\hline$\varepsilon$ & 0.64 & 0.75 & 0.64 & 0.75 \\
\hline
\end{tabular}

tration were made to construct the calibration curve between the blue intensity and its corresponding concentration.

Fig. (4) gives the unique relationship between the blue intensity and concentration, which can be used to determine the local solute concentration of the entire flow field within the test section of the channel.

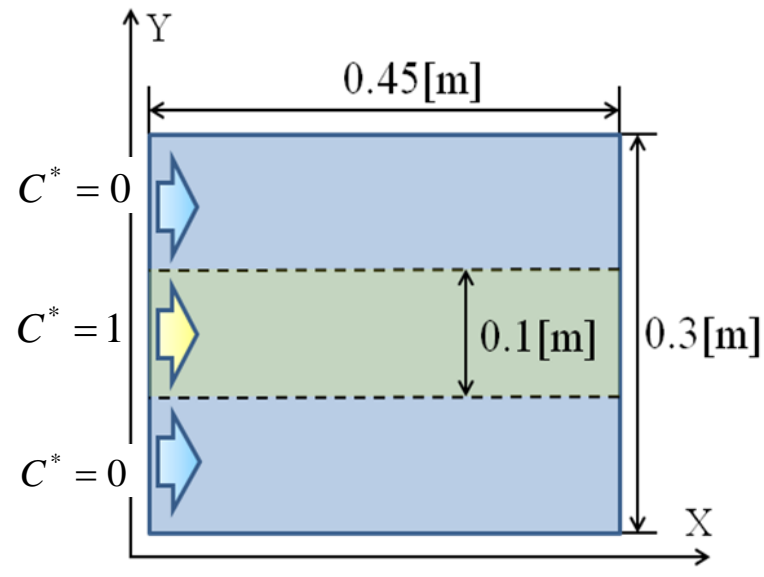

Fig. (3). Monitored section. 


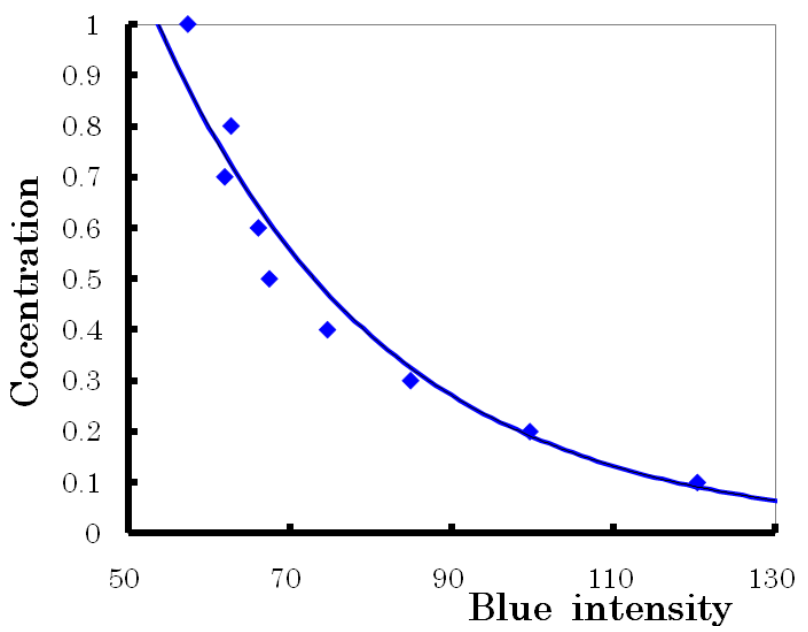

Fig. (4). Calibration curve.

A typical image obtained by the present visualization procedure is presented in Fig. $(\mathbf{5}(\mathbf{a}))$. The blue intensity of this image was extracted and transformed to show the concentrations within pores, as in Fig. $(\mathbf{5}(\mathbf{b}))$. Then, the Kernel method was exploited to obtain the macroscopic concentration field as shown in Fig. (5(c)).

\section{Macroscopic Numerical Model and Determination of Dispersion}

A macroscopic numerical model with an adjustable dispersion coefficient is used to predict the macroscopic concentration field. The resulting macroscopic concentration fields obtained with different values of dispersion coefficient are compared against the macroscopic concentration field obtained from the visualization, as shown in Fig. $(\mathbf{5}(\mathbf{c}))$. The dispersion coefficient may readily be determined from such a comparison that the predicted concentration field agrees well with the measured concentration field.

The macroscopic numerical model based on the volume averaged governing equation is given by

$\frac{\partial u_{j} C}{\partial x_{j}}=\left(\varepsilon K_{m}+K_{d i S_{Y Y}}\right) \frac{\partial}{\partial x_{j}}\left(\frac{\partial C}{\partial x_{j}}\right)$

where $K_{d i s_{Y Y}}$ is the adjustable dispersion coefficient. The molecular diffusivity of the fluorescein sodium solution $K_{m}$ is set to $4.5 \times 10^{-10}\left[\mathrm{~m}^{2} / \mathrm{s}\right][11]$.

Numerical computations were carried out for the solute diffusing flow in the shallow channel of porous structure using a standard numerical scheme with corresponding boundary conditions. Figs. (6(a), (b) and (c)) show such concentration fields obtained with assigning different values of $K_{d i s_{Y Y}}$. An increase in $K_{d i_{Y Y}}$ naturally results in widening the colored stream as mechanical dispersion becomes more significant downstream.

In order to determine the value of the dispersion coefficient, we shall focus on the concentration profiles at several axial stations, as indicated in Figs. (7(a) and (b)). Measured and predicted profiles of concentration are presented together in Fig. $(7(\mathbf{c}))$. This figure shows that the measured concentrations drop suddenly along the concentration edges, while

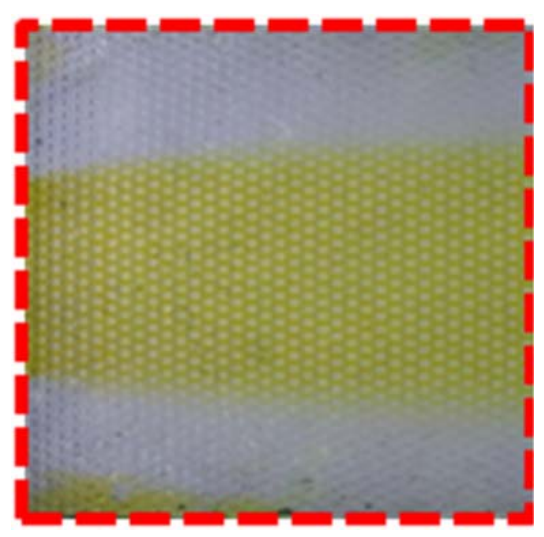

(a) Photographic image

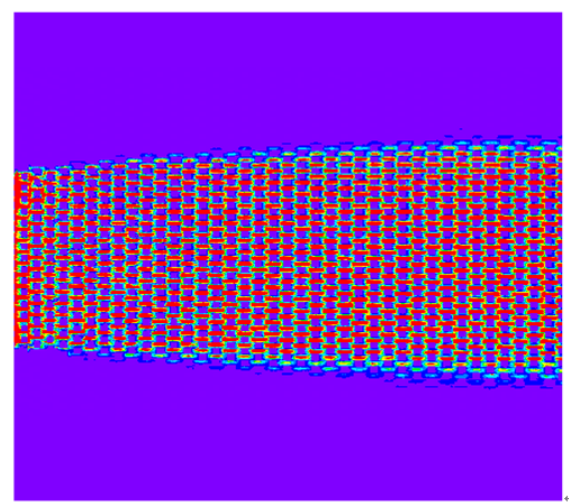

(b) Concentration within pores

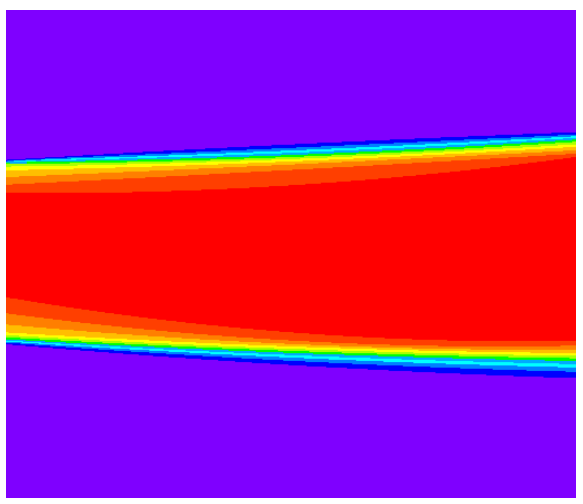

(c) Macroscopic concentration (Kernel method)

Fig. (5). Image processing $\left(\mathrm{Pe}_{\mathrm{D}}=3.0 \times 10^{5} \varepsilon=0.64 \mathrm{D} / \mathrm{H}=0.6\right)$.

the predicted concentrations drop somewhat more gradually to the level of the adjacent water streams. This discrepancy may be attributed to the fact that the isotropic tensor of the dispersion coefficient was used in the numerical model as given by Equation (1). Note that the longitudinal dispersion component is about 20 times larger than the transverse one. Suppression of the mechanical dispersion in the longitudinal direction may have enhanced the mechanical dispersion in the transverse direction. Such numerical verification is underway. Meanwhile, we shall resort to the present isotropic dispersion model and determine the transverse dispersion coefficient by fitting the numerical results based on the macroscopic model to the experimental results based on the concentration visualization in such a way to minimize the differ- 

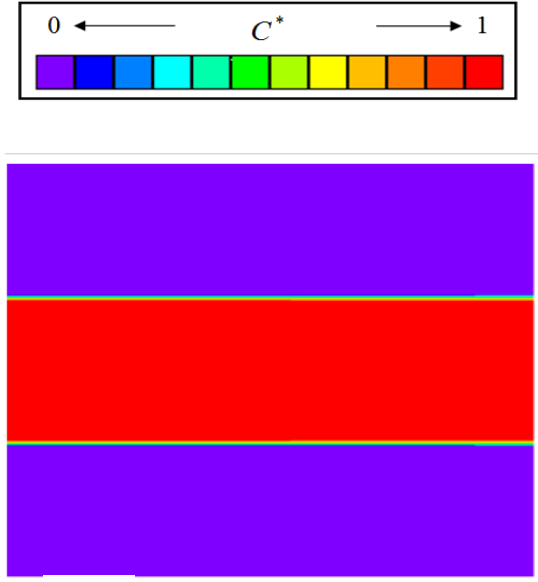

(a) $\mathrm{K}_{\text {disyy }}=1.0 \times 10^{4}$

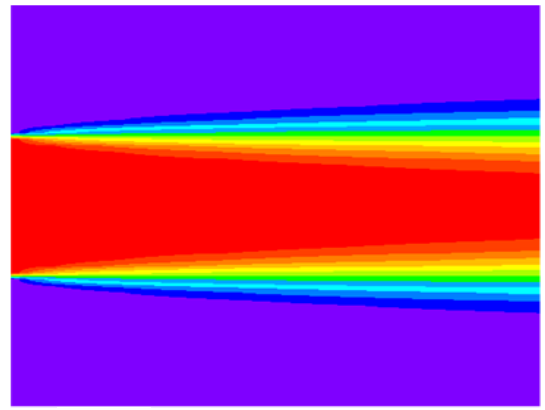

(b) $\mathrm{K}_{\text {disyy }}=5.0 \times 10^{5}$

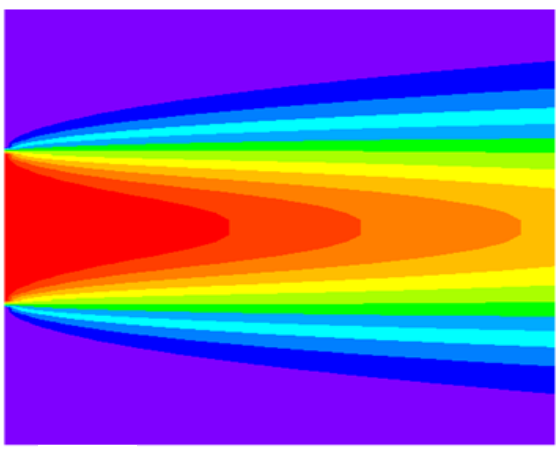

(c) $\mathrm{K}_{\text {disyy }}=5.0 \times 10^{6}$

Fig. (6). Numerical simulation results $\left(\mathrm{Pe}_{\mathrm{D}}=3.0 \times 10^{5} \quad \varepsilon=0.64\right.$ $\mathrm{D} / \mathrm{H}=0.6)$.

ence between the measure and predicted profiles at the selected axial stations.

The values of the dispersion coefficient obtained by this fitting procedure are presented in Figs. (8(a) and (b)) for the cases of $\varepsilon=0.64$ and 0.75 , respectively. In the same figure, our numerical results obtained from two-dimensional direct numerical simulations are presented for comparison. The present direct numerical simulation results for the case of the staggered arrangement are found to follow Equation (2) faithfully, which is given in terms of the ratio of the dispersion coefficient to the molecular diffusion coefficient as a function of the Peclet number:
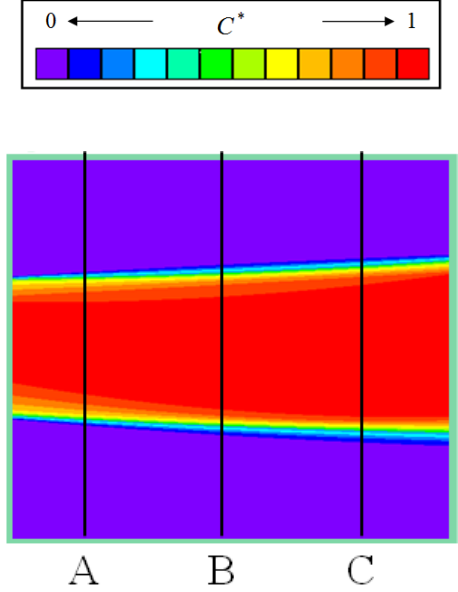

(a) Image from the experiment

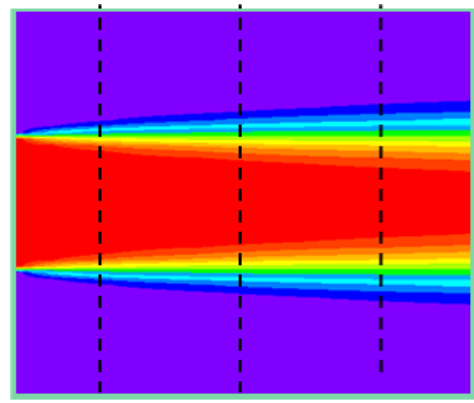

A $\quad$ B

C

(b) Image from the numerical model

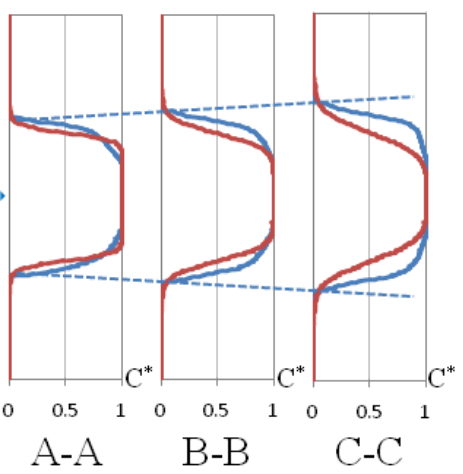

(c) Concentration profiles

Fig. (7). Comparison of concentration profiles $\left(\mathrm{Pe}_{\mathrm{D}}=3.0 \times 10^{5} \varepsilon=0.64\right.$ $\mathrm{D} / \mathrm{H}=0.6)$.

$\frac{K_{d s_{x}}}{K_{m}}=0.052(1-\varepsilon)^{1 / 2} P e_{D}$

The foregoing equation has been derived by Kuwahara et al. [9] by correlating their two dimensional direct numerical simulation results based on the two-dimensional periodic structure model of square rods.

The direct numerical simulations give fair agreement with the present experiments based on the visualization, although it tends to underestimate the level of the dispersion coefficient for the case of staggered arrangement $\varepsilon=0.64$. 


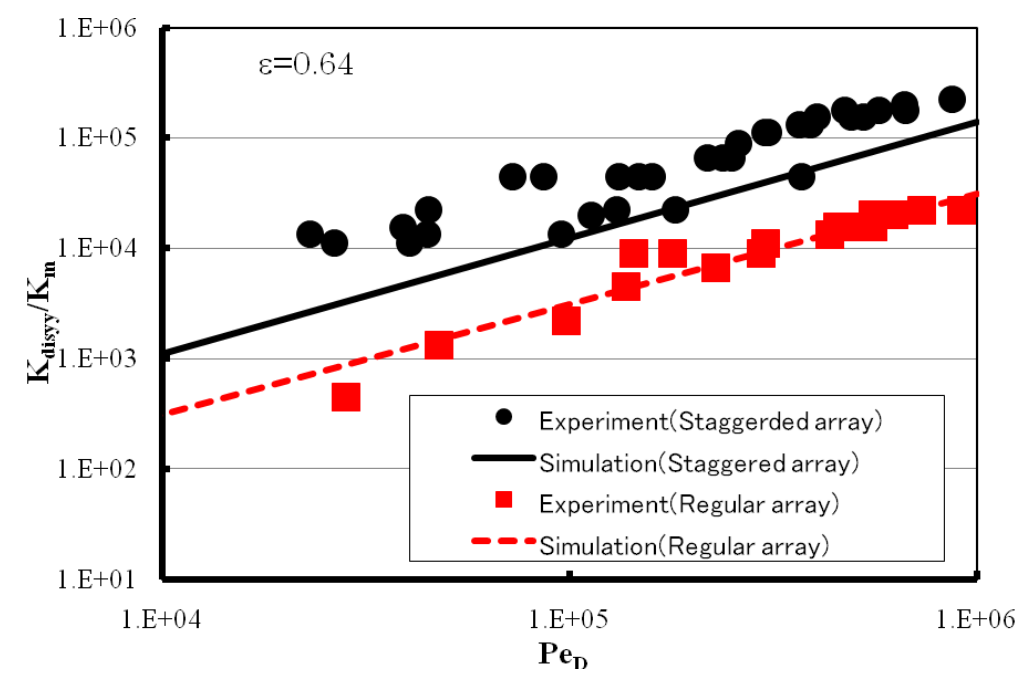

(a) $\varepsilon=0.64$

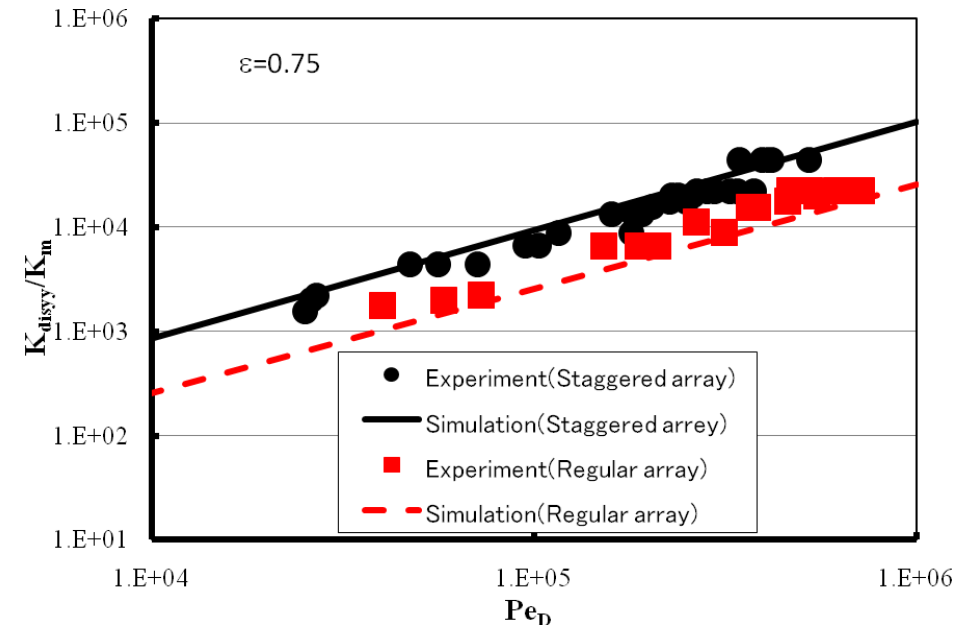

(b) $\varepsilon=0.75$

Fig. (8). Comparison of the experiments and predictions.

This underestimation indicates that the effects of the mechanical dispersion resulting from the presence of upper and lower walls of the channel on the total dispersion coefficient are quite significant. More over, comparison of Figs. (8(a) and (b)) reveals that the direct numerical simulations are incapable of describing rather significant effects of the porosity on the dispersion coefficient.

As, expected, the figures clearly shows that the mechanical dispersion coefficient for the case of staggered arrangement is much higher that that for the case of regular arrangement. Thus, the difference in the porous structure must be taken into account when estimating the dispersion coefficient. As the figures indicate, the present three-dimensional direct numerical simulation is quite effective for predicting the level of the dispersion coefficients.

Fully three-dimensional calculations have been also carried out only to capture the velocity field in a pore. Such preliminary three-dimensional velocity field is presented in Fig. (9). Upon faithfully describing the present porous struc- ture, three-dimensional direct numerical simulations will be carried out to determine the thermal dispersion coefficients in near future.

\section{CONCLUSIONS}

The study of mechanical dispersion in porous media was carried out using a concentration visualization technique with a dilute fluorescein sodium solution. Shallow channels with square blocks in regular and staggered arrangements were constructed to investigate the mechanical dispersion within fluid-saturated porous media. The technique is based on the correlation between the color intensity of blue and the concentration of fluorescein sodium. The dispersion coefficient was determined by fitting the numerical results based on the macroscopic numerical model to the experimentally obtained macroscopic concentration profiles. The direct numerical simulations were also carried out using periodic boundary conditions. The prediction level of the dispersion coefficients agrees well with that of the experiment based on the visualization technique. 


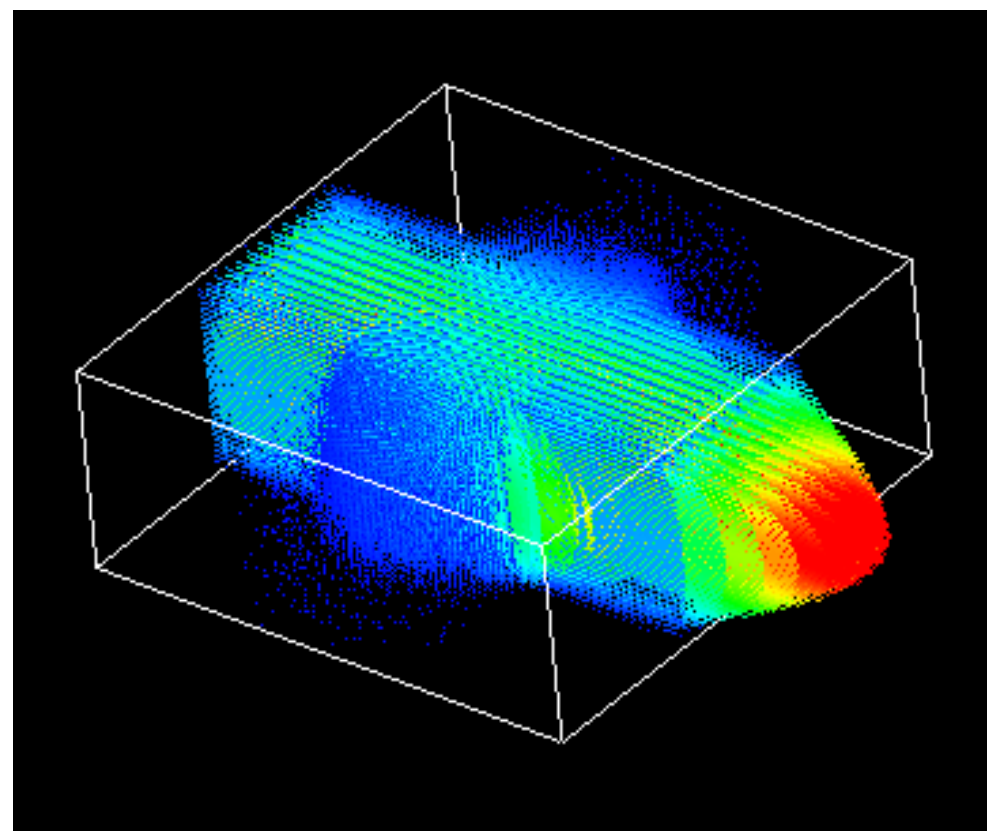

Fig. (9). Predicted velocity field in a pore (Regular array $\varepsilon=0.64$ ).

\section{NOMENCLATURE}

$\begin{array}{lll}C & = & \text { Molar concentration } \\ D & = & \text { Size of obstacle } \\ H & = & \text { Transverse length scale of unit cell } \\ K & = & \text { Mass diffusivity } \\ P e & = & \text { Peclet Number } \\ u_{i} & = & \text { Velocity vector } \\ W & = & \text { Longitudinal of unit cell } \\ x, y & = & \text { Cartesian coordinates }\end{array}$

\section{Greek Symbols}

$\varepsilon \quad$ Porosity

\section{Subscripts}

$\begin{array}{lll}m & = & \text { Molar diffusion } \\ \text { dis } & = & \text { Dispersion }\end{array}$

\section{REFERENCES}

[1] N. Wakao, and S. Kaguei, Heat and Mass Transfer in Packed Beds, Gordon and Breach: New York, 1982.
[2] S. Yagi, D. Kunii, and N. Wakao, "Studies on axial effective thermal conductivities in packed beds", AIChE Journal, vol. 6, pp. 543-546, 1960.

[3] GI. Taylor, "Dispersion of solute matter in solvent flowing slowly through a tube", Proc. Roy. Soc. London, vol. 15, pp. 1787-1806, 1953.

[4] R. Aris, "On the dispersion of a solute in a fluid flowing through a tube", Proc. Roy. Soc. London, vol. 235, pp. 67-77, 1956.

[5] DL. Koch, and JF. Brady, "Dispersion in fixed beds", Journal of Fluid Mechanics, vol. 154, pp. 399-427, 1985.

[6] NW. Han, J. Bhakta, and RG. Carbonell, "Longitudinal and lateral dispersion in packed beds: Effect of column length and particle size distribution", AIChE Journal, vol. 31, pp. 277-288, 1985.

[7] D. Vortmeyer, "Axial heat dispersion in packed beds", Chemical Engineering Science, vol. 30, pp. 999-1001, 1975.

[8] M. Kaviany, Principles of Heat Transfer in Porous Media, Springer-Verlag: New York, 1991, pp. 115-151.

[9] F. Kuwahara, A. Nakayama, and H. Koyama, "A numerical study of thermal dispersion in porous media", Trans. ASME Journal of Heat Transfer, vol. 118, pp. 756-761, 1996.

[10] F. Kuwahara, and A. Nakayama, "Numerical determination of thermal dispersion coefficients using a periodic porous structure", Trans. ASME Journal of Heat Transfer, vol. 121, pp. 160-163, 1999.

[11] Y. Masuda, T. Ohe, T. Yoshida, K. Nosita, K. Kato, S. Nagasaki, K. Amano, K. Futakuchi and T. Kaneko, "Measurement of both diffusion and distribution coefficients of some chemical tracers migrated in granite rock formation by adopting the micro-flow channel method", Trans. AESJ J.NUCE, vol. 16, pp. 3-16, 2009.

(C) Kuwahara et al.; Licensee Bentham Open.

This is an open access article licensed under the terms of the Creative Commons Attribution Non-Commercial License (http://creativecommons.org/licenses/by-nc/3.0/) which permits unrestricted, non-commercial use, distribution and reproduction in any medium, provided the work is properly cited. 